\title{
ON THE "VACUUM" DAM-BREAK PROBLEM: EXACT SOLUTIONS AND THEIR LONG TIME ASYMPTOTICS*
}

\author{
ROBERTO CAMASSA ${ }^{\dagger}$, GREGORIO FALQUI $^{\ddagger}$, GIOVANNI ORTENZI $^{\ddagger}$, \\ MARCO PEDRONI§${ }^{\S}$ AND GIUSEPPE PITTON
}

\begin{abstract}
The classical dam-break problem for the shallow water system with a dry/vacuum downstream state is revisited in the context of exact solutions which generalize the Riemann setup of a Heaviside jump between constant states to continuous initial data. Two main setups are considered, chosen to illustrate how local properties of the dependent variables at the vacuum point influence the evolution over different time scales. For the first case, the elevation (density) variable is initially continuous but not differentiable at the dry (vacuum) point: no gradient catastrophe develops in this variable, with the time evolution eventually merging into a single (shifted) Stoker wave. Conversely, for the second case, the elevation joins the dry state with vanishing first derivative and a curvature jump: for an instant in time a global gradient catastrophe at the fixed contact point forms and immediately evolves as a Stoker parabolic simple wave, allowing the contact point to split into two moving points, one at the dry bed and one at a fixed elevation, where curvature singularities persist at all times. Although in both cases shocks develop for the velocity field, these are nongeneric, in that, in contrast to the usual case, infinitely many conservation laws are satisfied at all times. Long time evolution is further analyzed with the help of new stretched "unfolding" variables to extract the details of the asymptotic approach to a rarefaction Stoker-like wave.
\end{abstract}

Key words. conservation laws, shallow water dynamics, weak solutions, vacuum states

AMS subject classifications. 35Q35, 35L67, 76B15

DOI. $10.1137 / 19 \mathrm{M} 1266836$

1. Introduction. The classical gas-dynamics Riemann problem in the presence of a vacuum state, and its hydrodynamic version for the so-called shallow water or Airy's system,

$$
u_{t}+u u_{x}+\eta_{x}=0, \quad \eta_{t}+(u \eta)_{x}=0,
$$

is a challenging topic that has recently received renewed attention in the literature (see, e.g., $[4,5,6,10,11,19]$ ). One of the main difficulties stems from the result [15] that no shocks can propagate into a vacuum/dry state, as the appropriate jump conditions cannot be satisfied at the shock. This is in contrast with its rarefaction wave counterpart, which of course is not subject to such conditions, and the corresponding dynamics can be analyzed in general [13, 15]. With this in mind, the study of classes of initial data that lead to closed form solutions can shed some light on the range of possible dynamics supported by the evolution equation and stimulate

\footnotetext{
${ }^{*}$ Received by the editors June 6, 2019; accepted for publication (in revised form) October 3, 2019; published electronically January 2, 2020.

https://doi.org/10.1137/19M1266836

Funding: This work was supported by the National Science Foundation under grants RTG DMS-0943851, CMG ARC-1025523, DMS-1009750, and DMS-1517879; the Office of Naval Research under grants N00014-18-1-2490 and DURIP N00014-12-1-0749; ERC Consolidator Grant 682603; and grant H2020-MSCA-RISE-2017 project 778010 IPaDEGAN.

${ }^{\dagger}$ Department of Mathematics, Carolina Center for Interdisciplinary Applied Mathematics University of North Carolina at Chapel Hill, Chapel Hill, North Carolina, 27599 (camassa@email.unc.edu).

${ }^{\ddagger}$ Dipartimento di Matematica e Applicazioni, Universita’ di Milano-Bicocca, Milano, Italy 20125 (gregorio.falqui@unimib.it, giovanni.ortenzi@unimib.it).

${ }^{\S}$ Dipartimento di Ingegneria Gestionale, dell'Informazione e della Produzione, Universita' di Bergamo-Dalmine, Dalmine (BG), Italy 24044 (marco.pedroni@unibg.it).

\Department of Mathematics, Imperial College, London, UK (g.pitton@imperial.ac.uk).
} 
general proofs and extensions of existing results in the literature (see, e.g., [6, 11, 19] in the context of the so-called physical vacuum) including higher dimensional settings. This is the focus of our paper.

We consider piecewise smooth initial data on the real line with a vacuum region corresponding to $x<0$, connecting continuously to "density" (actually the fluid layer thickness in the hydrodynamic interpretation of (1.1)) $\eta \geq 0$ for $x>0$. While the velocity $u$ for the vacuum region clearly has no physical meaning, mathematically the manifold $\eta=0$ is an invariant reduction of the system to the Hopf equation

$$
u_{t}+u u_{x}=0 .
$$

For the class of solution we consider in this study, with vanishing initial velocity $u(x, 0)=0$, continuous dependence on the initial data can in fact lead to a jump of this field across the location where $\eta(x, t)=0$, i.e., a shock for the variable $u$ alone while continuity of $\eta$ is maintained. This situation is different from the most common case studied in the literature, where jumps of the dependent variables occur simultaneously. The standard Rankine-Hugoniot conditions are satisfied for the cases we consider due to the vanishing of $\eta$ at the shock location; thus the interpretation of the resulting weak solutions as those with propagating shocks is justified. While we focus on a particular set of initial data, our results apply locally to a larger class, where $\eta$ and $u$ admit a Taylor series expansion in the (right) neighborhood of the contact point where $\eta=0$.

This paper is organized as follows. After a brief review of the Riemann problem in section 2, we define our "dam-break" classes in section 3 and 4, respectively, for the continuous and differentiable cases. The dynamics supported by the latter setup is further illustrated by a variant of the Riemann problem, obtained by a version of the backward in time prolongation of the dam-break initial data. Next, borrowing from a recent result [2] in a different context, we introduce "unfolding" or stretched variables, which can be used to follow the long time asymptotics of both solution classes. Numerical simulations with a general purpose weighted essentially nonoscillatory (WENO) algorithm both illustrate the evolution as predicted by the exact solutions and highlight the limitations of this scheme in the presence of a vacuum state.

2. An overview of the classical Riemann problem. In this section we briefly recall the dam-break solution (see, e.g., [21]) of the shallow water, or Airy's, model

$$
u_{t}+u u_{x}+\eta_{x}=0, \quad \eta_{t}+(u \eta)_{x}=0,
$$

where, in appropriately scaled variables, $\eta(x, t)$ represents the surface height and $u(x, t)$ is the horizontal layer-averaged velocity at location $x \in \mathbb{R}$ and time $t \in \mathbb{R}^{+}$. The initial condition representing a dam at the origin filled to a height $Q$ is

$$
\eta(x, 0)=\left\{\begin{array}{ll}
0, & x<0, \\
Q, & x \geq 0,
\end{array} \quad u(x, 0)=0 .\right.
$$

The corresponding solution satisfies $\eta(p(t), t)=0$ and $\eta(b(t), t)=Q$, at moving points $p(t)$ and $b(t)$, and is constant outside the interval $[p(t), b(t)]$. Inside this interval, the solution is given by a so-called simple wave, meaning that one of the two Riemann 
invariants is the same constant over the whole interval. Indeed, recall that the characteristics of the system (1.1) are the solution of the ODEs

$$
\dot{x}_{ \pm}=\lambda_{ \pm}(\eta, u), \quad \text { where } \lambda_{ \pm}(\eta, u)=u \pm \sqrt{\eta},
$$

and the so-called Riemann invariants $R_{ \pm}(\eta, u)=u \pm 2 \sqrt{\eta}$ are constant along the corresponding characteristics. In other words, system (1.1) is equivalent (in the physical region of interest $\eta \geq 0$ ) to

$$
\partial_{t} R_{ \pm}+\lambda_{ \pm}\left(R_{+}, R_{-}\right) \partial_{x} R_{ \pm}=0 .
$$

Following the negative characteristic starting from points $\left(x_{0}, 0\right)$, with $x_{0}>0$, the Riemann invariant $R_{-}$is easily seen to assume the constant value $-2 \sqrt{Q}$ in the simple wave region $S=\{(x, t) \mid p(t)<x<b(t), t \geq 0\}$, where the field $\eta$ and $u$ are functionally related by $u(x, t)-2 \sqrt{\eta(x, t)}=-2 \sqrt{Q}$. It is easy to check that the solution in the region $S$ is given by the simple rarefaction wave

$$
\eta=\frac{1}{9}\left(\frac{x}{t}+2 \sqrt{Q}\right)^{2}, \quad u=\frac{2}{3}\left(\frac{x}{t}-\sqrt{Q}\right),
$$

so that $p(t)=-2 t \sqrt{Q}$ and $b(t)=t \sqrt{Q}$. The whole dam-break solution can then be defined as

$$
\begin{gathered}
\eta(x, t)=\left\{\begin{array}{cl}
0, & x<-2 t \sqrt{Q}, \\
\frac{1}{9}\left(\frac{x}{t}+2 \sqrt{Q}\right)^{2}, & -2 t \sqrt{Q} \leq x \leq t \sqrt{Q}, \\
\sqrt{Q}, & x>t \sqrt{Q},
\end{array}\right. \\
u(x, t)=\left\{\begin{array}{cc}
0, & x<-2 t \sqrt{Q}, \\
\frac{2}{3}\left(\frac{x}{t}-\sqrt{Q}\right), & -2 t \sqrt{Q} \leq x \leq t \sqrt{Q}, \\
0, & x>t \sqrt{Q} .
\end{array}\right.
\end{gathered}
$$

It is interesting to note (see, e.g., [21]) that the layer thickness remains constant at the origin, $\eta(0, t)=4 Q / 9$, for all $t>0$, that is, the interface always passes through the point $(0,4 Q / 9)$.

With definition (2.6) for the weak solution of system (1.1), and in particular having chosen the velocity $u=0$ in the region $x<p(t)$, it is immediate to check that the jump from $u=0$ to $u=-2 \sqrt{Q}$ at $x=p(t)$ consistently defines a shock velocity as

$$
\dot{p}=\frac{[\eta u]}{[\eta]} \equiv \lim _{\epsilon \rightarrow 0} \frac{\eta(p(t)+\epsilon, t) u(p(t)+\epsilon, t)-\eta(p(t)-\epsilon, t) u(p(t)-\epsilon, t)}{\eta(p(t)+\epsilon, t)-\eta(p(t)-\epsilon, t)}=-2 \sqrt{Q},
$$

i.e., this shock moves with the fluid's velocity, which is well defined when $\eta>0$. For $x<p(t)$, the choice $u=0$, which leads to a jump in this variable, is somewhat arbitrary from a physical perspective, since no fluid is present to the left of the moving point $p(t)$. However, from a mathematical viewpoint, setting $\eta=0$ reduces system (1.1) to the Hopf equation $u_{t}+u u_{x}=0$ for $u$, and the choice of $u$ in the region $x<p(t)$ should be made consistently with a solution to this equation, e.g., for any 

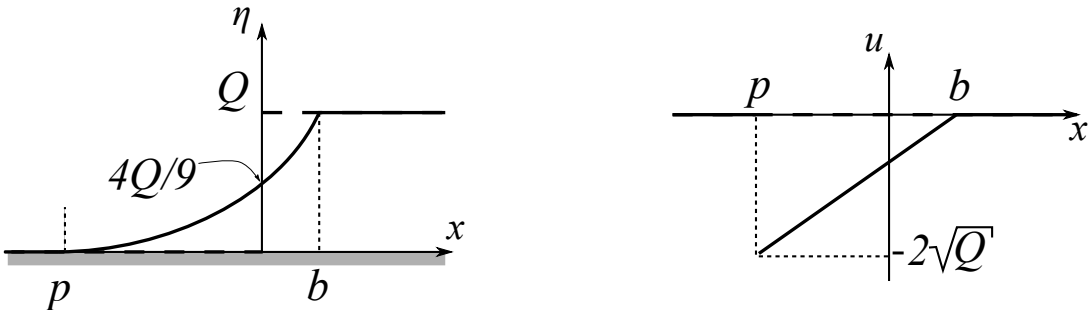

FIG. 1. Sketch of the evolution of $\eta(x, t)$ and $u(x, t)$ starting from the initial data (2.2). The relevant $x$-values are $p(t)=-2 t \sqrt{Q}$ and $b(t)=t \sqrt{Q}$. The dashed lines are the initial data while the bold lines are their evolution.

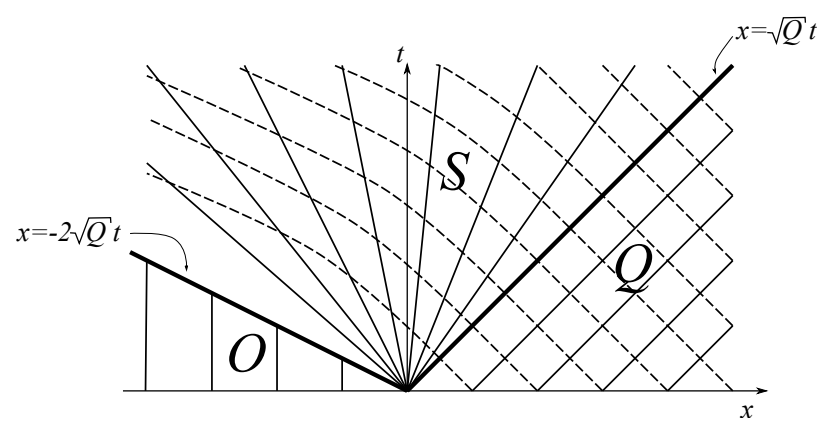

FIG. 2. Positive (continuous line) and negative (dashed line) characteristics for the solution (2.6). The three regions, from right to left, labelled $Q, S$, and $O$, correspond to, respectively, the rest region $x>b(t)$ where $\eta=Q$ and $u=0$, the simple wave region $S$ between the last positive characteristic $x=b(t)$ and the contact point $x=p(t)$ where $\eta=0$, and the parabolic region $O$, where only one family of characteristics can be defined.

constant value for $u$. In particular, the choice $u=-2 \sqrt{Q}$ for $x<p(t)$, which is the one made in [21], is also available and makes the $u$-field continuous, thereby eliminating the interpretation of the moving point $x=p(t)$ as a shock location. However, note that this would imply an initial discontinuity in the velocity field, whereby the second equation in $(2.2)$ would be replaced by $u(x, 0)=-2 \sqrt{Q} H(-x)$, where $H$ is the Heaviside step function. Similarly, the analogue of the jump condition (2.7) for the momentum $m \equiv \eta u$ is satisfied with any choice $u=$ const. for $x<p(t)$, and in fact for infinitely many conserved quantities; see below. For $\eta=0$, even if system (1.1) has coincident characteristics eigenvalues $\lambda_{+}=\lambda_{-}$, and thus it is degenerate of parabolic kind in this region, such shock conditions can be still interpreted as local conservation laws. The solution (2.6) is depicted in Figure 1, and the corresponding characteristics are shown in Figure 2.

Remark 2.1. As is well known, not all quantities conserved by strong solutions of system (1.1) are still conserved by weak solutions. In the presence of shocks, selecting which conservation laws are to be enforced determines the shock speed. For instance, with the conserved density $u$ and its related current $\eta+u^{2} / 2$ the shock velocity is $-\sqrt{Q}$, which does not coincide with $\dot{p}=-2 \sqrt{Q}$ given by (2.7). However, in the presence of a vacuum state with continuous $\eta$, an infinite number of conserved quantities survives. A simple way to prove this peculiar property exploits the integrability structure of system (1.1). By means of the classical Lenard-Magri recursion for the bi-Hamiltonian structure of the model $[1,16]$, one finds a one-parameter family of conserved densities, 


$$
h(u, \eta ; z)=-\sqrt{\frac{(u-z)^{2}}{4}-\eta}+\frac{z-u}{2},
$$

whose associated current generator is

$$
H(u, \eta ; z)=\frac{z+u}{2} h(u, \eta ; z)-\frac{1}{2} \eta .
$$

In the limit $z \rightarrow+\infty$, a countable number of conserved densities arises:

$$
h(u, \eta ; z)=\frac{\eta}{z}+\frac{\eta u}{z^{2}}+\frac{\eta^{2}+\eta u^{2}}{z^{3}}+\frac{\eta^{2} u+\eta u^{3}}{z^{4}}+\frac{2 \eta^{3}+6 \eta^{2} u^{2}+\eta u^{4}}{z^{5}}+\cdots .
$$

The shock velocity $v_{s}$ for an arbitrary fixed value of $z$ is

$$
v_{s}(z)=\frac{H_{R}-H_{L}}{h_{R}-h_{L}},
$$

where $f_{R}$ and $f_{L}$ stand for the values of $f$ on the right and on the left of $p(t)=$ $-2 \sqrt{Q} t$. This reduces to

$$
\begin{aligned}
v_{s}(z) & =\lim _{\eta_{R} \rightarrow 0} \frac{H\left(-2 \sqrt{Q}, \eta_{R} ; z\right)-H(0,0 ; z)}{h\left(-2 \sqrt{Q}, \eta_{R} ; z\right)-h(0,0 ; z)} \\
& =\lim _{\eta_{R} \rightarrow 0} \frac{H\left(-2 \sqrt{Q}, \eta_{R} ; z\right)}{h\left(-2 \sqrt{Q}, \eta_{R} ; z\right)}=-2 \sqrt{Q}
\end{aligned}
$$

for all $z$. In particular, each term of series (2.10) (and in particular the energy, which is the $z^{-3}$ term of (2.10)) gives rise to a conserved quantity even in the presence of velocity shocks located at points where $\eta$ joins continuously $\eta=0$.

Having reviewed the essential elements of the classical dam-break problem, we now look at extensions which can still lead to closed form solutions. While still special, these solutions nonetheless illustrate how the simplicity of the classical dam-break problem above can hide fundamental differences with the more generic case, where one simple (rarefaction) wave cannot account for the global structure of the solution.

3. Dam-break: the linear connection case. Outside of the simple wave class, exact solutions of system (1.1) can always be sought in Taylor series form with time dependent coefficients which would then satisfy an infinite system of ODEs. A notable class of solutions in this form consists of a reduction to a finite number of ODEs, whose solutions then determine the whole (possibly infinite) hierarchy of coefficients. Hence the special solutions generated this way can be viewed as corresponding to "finite degree of freedom" reductions of the dynamics supported by system (1.1) [2]. The simplest case of this reduction is that of linear and quadratic polynomials, where all higher order coefficients of the Taylor series can be taken to be zero, so that closed form solutions can actually be computed. While this strategy is essentially local, since in general it does not support physical relevant boundary conditions for the system (1.1), it does reduce this PDE to a system of ODEs governing the evolution of the time dependent coefficients. It has been proposed in the context of shallow water models in [17] and for the compressible Euler system, subject to a regularization by linear damping, in [14]. 
3.1. Linear core exact solution. The shallow water model (1.1) admits linear solutions

$$
\eta=\beta(t) x+\mu(t), \quad u=\nu(t) x+\kappa(t),
$$

where the coefficients of the straight lines are suitable functions of $t$, solving the triangular ODE system

$$
\dot{\nu}+\nu^{2}=0, \quad \dot{\beta}+2 \nu \beta=0, \quad \dot{\kappa}+\nu \kappa+\beta=0, \quad \dot{\mu}+\nu \mu+\beta \kappa=0
$$

with initial data

$$
\nu(0)=\nu_{0}, \quad \beta(0)=\beta_{0}, \quad \kappa(0)=\kappa_{0}, \quad \mu(0)=\mu_{0} .
$$

After a translation of the $x$-axis, we can set $\mu_{0}=0$. Moreover, up to sign change reflections of $x$ and $u$, we can always assume $\beta_{0}>0$. If the initial velocities vanish, i.e., $\nu_{0}=0$ and $\kappa_{0}=0$, then the solution is easily computed:

$$
\eta=\beta_{0} x+\frac{1}{2} \beta_{0}^{2} t^{2}, \quad u=-\beta_{0} t
$$

The characteristics passing through $\left(x_{0}, 0\right)$ for $x_{0} \geq 0$ are determined by the corresponding Riemann invariant

$$
R_{ \pm}\left(x_{ \pm}(t), t\right)=\beta_{0} t \pm 2 \sqrt{\beta_{0} x_{ \pm}(t)+\frac{1}{2} \beta_{0}^{2} t^{2}}= \pm 2 \sqrt{\beta_{0} x_{0}}
$$

or, explicitly,

$$
x_{ \pm}\left(t ; x_{0}\right)=x_{0} \pm t \sqrt{\beta_{0} x_{0}}-\frac{1}{4} \beta_{0} t^{2} .
$$

Remark 3.1. It is clear from the right-hand side of (3.5) that these curves exist only in the region $x \geq-\frac{1}{2} \beta_{0} t^{2}$. This is in agreement with the fact that $\eta\left(-\beta_{0} t^{2} / 2, t\right)=$ 0 , since $\eta=0$ separates the hyperbolic and the elliptic region of system (1.1). Hence, the positive characteristics (labeled by "+" in (3.6)) are defined for all $t \geq 0$, while the negative ones (labelled by "-") are defined for $0 \leq t \leq 2 \sqrt{x_{0} / \beta_{0}}$ and cease to exist at the curve $x=-\beta_{0} t^{2} / 2$. As shown explicitly by this relation, this case falls into the so-called physical vacuum class, as the dry/vacuum point moves with nonzero acceleration $\dot{p}=-\beta_{0}$ t.

Remark 3.2. The two curves $x_{-}\left(t ; x_{0}\right)$ and $x=-\beta_{0} t^{2} / 2$ are tangent at the intersection point $\left(-2 x_{0}, 2 \sqrt{x_{0} / \beta_{0}}\right)$, so that the latter curve can also be viewed as the envelope of these characteristics. The curve $x_{-}\left(t ; x_{0}\right)$ for $t \geq 2 \sqrt{x_{0} / \beta_{0}}$ is in fact the positive characteristic emanating from the tangent point. This nonuniqueness of solutions for the characteristic equations (2.3) is to be expected, as $\lambda_{ \pm}$contain a square root of dependent variables whereby Lipshitz continuity fails at the tangent point. In the hodograph plane $(u, \eta)$, characteristics trace arcs of parabolae $u \pm 2 \sqrt{\eta}=$ const., which have $\eta=0$ as their envelope (cf. (3.4)).

To compare the present case with that in our previous discussion for the classical dam-break solution, it is useful to note that the parabola $x=-\beta_{0} t^{2} / 2$ admits yet another characterization, namely, it can be viewed as location of the (degenerate) shock class mentioned above, whereby the velocity $u$ jumps while the layer thickness $\eta$ 


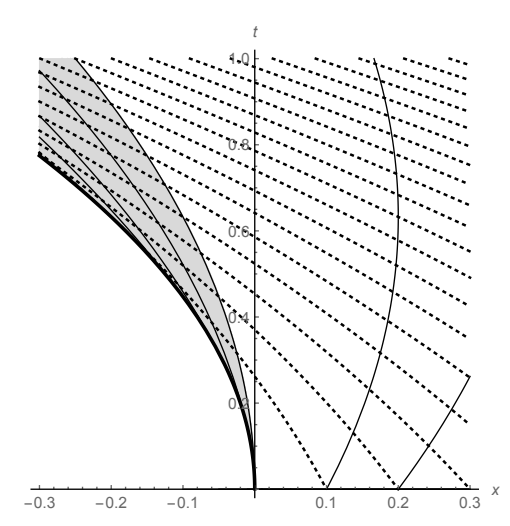

FIG. 3. Positive (continuous line) and negative (dashed line) characteristics for the solution (3.4) with $\beta_{0}=1$. The thick line is $x=-\frac{1}{2} \beta_{0} t^{2}$, where $\eta=0$. In the grey region, the positive characteristics emanate from $x=-\frac{1}{2} \beta_{0} t^{2}$, rather than $t=0$, and their analytical form coincides with that of the negative characteristics.

joins continuously the value $\eta=0$ where the system (1.1) becomes parabolic. Indeed, two characteristics with initial conditions $x_{0}$ and $x_{0}^{\prime}$ intersect, i.e.,

$$
x_{0} \pm t \sqrt{\beta_{0} x_{0}}-\frac{1}{4} \beta_{0} t^{2}=x_{0}^{\prime} \pm t \sqrt{\beta_{0} x_{0}^{\prime}}-\frac{1}{4} \beta_{0} t^{2},
$$

at time

$$
t=\mp\left(\sqrt{\frac{x_{0}^{\prime}}{\beta_{0}}}+\sqrt{\frac{x_{0}}{\beta_{0}}}\right) .
$$

Therefore the negative-class characteristics intersect at some positive time, which would imply, according to the general theory (see, e.g., [22]), that a shock needs to be fitted to maintain single-valuedness. The earliest shock time (for a single characteristic starting from $x_{0}$ ) is

$$
t\left(x_{0}\right)=2 \sqrt{\frac{x_{0}}{\beta_{0}}} .
$$

Eliminating the parameter $x_{0}$ by using this in (3.6), with the choice of negative sign, yields $x=-\beta_{0} t^{2} / 2$, which is therefore the location of intersections of characteristics of the same (negative) class. Notice that characteristics starting from base points $x_{0}^{\prime}<x_{0}$ cease to exist before $t=t\left(x_{0}\right)$. This behavior of characteristics is depicted in Figure 3 .

3.2. Piecewise linear-constant solution. The linear initial condition above for the fluid layer thickness $\eta$, and its $(\eta, u)$ evolution, can be made to satisfy physical boundary conditions as $x \rightarrow \infty$ by "splicing" it with constant values of the dependent variables. Thus, we now consider the evolution starting from rest with a dry region $\eta=0$ connected by a straight line to a constant height $\eta=Q$. This can be seen as an intermediate case between the classical Riemann dam-break problem and the parabolic case, to be discussed in the next section, in that the $\eta$-initial data are continuous but nondifferentiable at the origin, as opposed to discontinuous for the Riemann case and $C^{1}$ at the dry point for the parabolic case. 
The initial condition is

$$
\eta(x, 0)=\left\{\begin{array}{ll}
0, & x<0 \\
\beta_{0} x, & 0 \leq x \leq a_{0}, \\
Q, & x>a_{0},
\end{array} \quad u(x, 0)=0,\right.
$$

where $a_{0}=Q / \beta_{0}$. Given what we know about the linear solution core from the previous section, the solution is

$$
\begin{aligned}
& \eta(x, t)=\left\{\begin{array}{lr}
0, & x<p(t), \\
\beta_{0} x+\frac{1}{2} \beta_{0}^{2} t^{2}, & p(t) \leq x<a(t),
\end{array}\right. \\
& u(x, t)=\left\{\begin{array}{lr}
0, & x<p(t), \\
-\beta_{0} t, & p(t) \leq x<a(t),
\end{array}\right.
\end{aligned}
$$

where we have kept, by analogy with the Riemann case of section 2 , the notation $p(t)$ for the point where $\eta$ vanishes; thus at all times where the linear solution (3.4) exists, $p(t)=-\beta_{0} t^{2} / 2$. Here $a(t)$ is the moving point, to be determined, of the right end of the linear solution interval, so that $a(0)=a_{0}$. Note that singularities can develop to limit the time of solution existence, and the above solution may only exist for finite times. To find $a(t)$ we make the following ansatz,

$$
\begin{aligned}
\eta(x, t) & =\left(\beta_{0} x+\frac{1}{2} \beta_{0}^{2} t^{2}\right) \chi_{\mathcal{A}}(x)+N(x, t) \chi_{\mathcal{B}}(x)+Q H(x-b(t)), \\
u(x, t) & =-\beta_{0} t \chi_{\mathcal{A}}(x)+V(x, t) \chi_{\mathcal{B}}(x)
\end{aligned}
$$

where $N(x, t)$ and $V(x, t)$ are, respectively, the local surface and the velocity solutions of the Airy system (1.1) for $a(t)<x<b(t), \chi_{\mathcal{I}}(x)$ is the characteristic function of the interval $\mathcal{I}=\left(x_{1}, x_{2}\right) \subset \mathbb{R}$, that is,

$$
\chi_{\mathcal{I}}(x)=H\left(x-x_{1}\right)-H\left(x-x_{2}\right),
$$

with $H$ the Heaviside step function, $\mathcal{A}=(p(t), a(t))$, and $\mathcal{B}=(a(t), b(t))$. Here $b(t)$ is another moving point to be determined such that $b(0)=a_{0}$. Thus, we anticipate that the initial corner at $x=a_{0}$ can split into two corners supported at the moving points $a(t)$ and $b(t)$ where the solution joins continuously the linear core and the constant background, respectively, so that $(\eta, u)$, at least up to a possible gradient catastrophe time, is continuous at all points $x>p(t)$. Differentiating the Airy system with respect to $x$ yields

$$
u_{x t}+\left(\frac{u^{2}}{2}+\eta\right)_{x x}=0, \quad \eta_{x t}+(u \eta)_{x x}=0,
$$

and when the solution ansatz (3.12) is substituted in these equations, using the distributional relation

$$
\chi_{\mathcal{I}}^{\prime}=\delta\left(x-x_{1}\right)-\delta\left(x-x_{2}\right),
$$

three different kinds of distributions (Heaviside $H$, Dirac $-\delta$, and Dirac $-\delta^{\prime}$ ) appear and need to be separately balanced to find a solution in the sense of distributions. The 
terms involving Heaviside functions cancel out because (3.12) is a piecewise solution of the Airy system, and the same happens with the terms involving Dirac $-\delta^{\prime}$ because of the continuity of (3.12) for $x>p(t)$. Therefore the only terms governing the evolution of the points $a(t)$ and $b(t)$ come from the Dirac- $\delta$ 's, and read

$$
\begin{aligned}
& \left(\beta_{0}-N_{x}(a(t), t)\right)\left(\dot{a}(t)+\beta_{0} t\right)+V_{x}(a(t), t)\left(\beta_{0} a(t)+\frac{1}{2} \beta_{0}^{2} t^{2}\right)=0, \\
& V_{x}(a(t), t)\left(\dot{a}(t)+\beta_{0} t\right)+\beta_{0}-N_{x}(a(t), t)=0
\end{aligned}
$$

for the terms supported at $x=a(t)$ and

$$
N_{x}(b(t), t) \dot{b}(t)-Q V_{x}(b(t), t)=0, \quad V_{x}(b(t), t) \dot{b}(t)-N_{x}(b(t), t)=0
$$

for the terms supported at $b(t)$. If $N_{x}(b(t), t) V_{x}(b(t), t) \neq 0$, from system (3.16) it follows that $\dot{b}(t)^{2}=Q$, so that $\dot{b}(t)= \pm \sqrt{Q}$. The choice of the plus sign moves the point $b(t)$ along a right going characteristic; therefore we obtain

$$
b(t)=\frac{Q}{\beta_{0}}+t \sqrt{Q} .
$$

As to the evolution of $a(t)$, if $\left(\beta_{0}-N_{x}(a(t), t)\right) V_{x}(a(t), t) \neq 0$, from system (3.15) it follows that

$$
\left(\frac{d}{d t}\left(a(t)+\frac{1}{2} \beta_{0} t^{2}\right)\right)^{2}=\beta_{0}\left(a(t)+\frac{1}{2} \beta_{0} t^{2}\right) .
$$

Hence with the choice of negative sign for a left going characteristic, we have

$$
\frac{d}{d t}\left(a(t)+\frac{1}{2} \beta_{0} t^{2}\right)=-\sqrt{\beta_{0}\left(a(t)+\frac{1}{2} \beta_{0} t^{2}\right)},
$$

whose solution satisfying $a(0)=a_{0}=Q / \beta_{0}$ is easily seen to be

$$
a(t)=\frac{Q}{\beta_{0}}-t \sqrt{Q}-\frac{1}{4} \beta_{0} t^{2} .
$$

This solution shows that there exists a finite time $t_{c}=2 \sqrt{Q} / \beta_{0}$ when a collision $a(t)=$ $p(t)$ occurs. This corresponds to the time when the core straight line disappears, and the solution supported on the intermediate interval $\mathcal{B}$ connects the dry region to the left of $p(t)$ to the background quiescent state to the right of $b(t)$.

Remark 3.3. The above computation by distributional calculus shows explicitly how the classical result [22, p. 127] applies to corner points $x=0$ and $x=a_{0}$ moving along characteristics. However, it should be stressed that the point at $x=0$ behaves differently than its counterpart at $x=a_{0}$. In fact, the interpretation of $x=p(t)$ as an envelope of characteristics shows that a loss of regularity could be expected at this point, as characteristics of the same class cross along this curve. Accordingly, the continuity of the velocity component of system (1.1) can be expected to be lost at times $t>0$, unlike the case for the classical Riemann dam-break in section 2, where the dry region $\eta=0$ could be covered with a constant solution of the Hopf equation $u_{t}+u u_{x}=$ 0 to join continuously the value $u(p(t), t)=-2 \sqrt{Q}$. In particular, no splitting of the corner point initially at $x=0$ along two characteristics can be observed in the solution. 
From the results in [22], it follows that a necessary condition for a corner point initially at $x=\hat{x}$ to split into two points, each following a different characteristic, is that the characteristic eigenvalues are different, $\lambda_{-}(\eta(\hat{x}, 0), u(\hat{x}, 0)) \neq \lambda_{+}(\eta(\hat{x}, 0), u(\hat{x}, 0))$; while we do not pursue a full analysis of this point here, we conjecture that, to be sufficient, this condition has to be augmented by the requirement that both Riemann invariants have a discontinuous $(t$ - or $x$-)derivative at $(\hat{x}, 0)$. Thus, the initial corner at $x=0$, where $\eta=0$, does not separate into two points and remains at the bottom (i.e., its evolution is given by $x=p(t)=-\beta_{0} t^{2} / 2$ ) for the finite time $t<t_{c}$, whereas the second corner point initially at $x=a_{0}$ splits along the negative characteristic curve (3.20) (cf. the general expression (3.6)), and its positive counterpart (3.17) (associated with the constant solution $\eta=Q, u=0$ ), for all times $t>0$.

Collecting results (3.17) and (3.20) yields the explicit form of the piecewise solution for the intermediate times $t \in\left[0, t_{c}\right]$,

$$
\begin{gathered}
\eta(x, t)=\left\{\begin{array}{lr}
0, & x<p(t), \\
\beta_{0} x+\frac{1}{2} \beta_{0}^{2} t^{2}, & p(t) \leq x \leq a(t), \\
N(x, t), & a(t)<x \leq b(t), \\
Q, & x>b(t),
\end{array}\right. \\
u(x, t)=\left\{\begin{array}{lr}
0, & x<p(t), \\
-\beta_{0} t, & p(t) \leq x \leq a(t), \\
V(x, t), & a(t)<x \leq b(t), \\
0, & x>b(t),
\end{array}\right.
\end{gathered}
$$

where $N(x, t)$ and $V(x, t)$ are, respectively, an interface and a velocity field solving the system (1.1) in the time-varying interval $\mathcal{B}=(a(t), b(t))$. We will call the pair $(N, V)$, connecting the straight line with the constant height $Q$, the "shoulder" part of the solution. By the same argument used in section 2, one can show that the Riemann invariant $R_{-}(\eta, u)=u-2 \sqrt{\eta}$ is constant, $R_{-}(\eta, u)=-2 \sqrt{Q}$, when evaluated at $\eta=N(x, t)$ and $u=V(x, t)$, so that the shoulder corresponds to a simple wave solution. In the region $S$ where the shoulder is defined (see Figure 4), the positive characteristics are straight lines (starting from the curve $x=a(t)$ ). Indeed, the slope $\lambda_{+}=V+\sqrt{N}=\left(3 R_{+}+R_{-}\right) / 4$ of such a characteristic is invariant along the characteristic itself.

To compute $N(x, t)$ and $V(x, t)$ at a given point $(x, t) \in S$, denote by $\left(a\left(t_{0}\right), t_{0}\right)$ the point lying on the positive characteristic passing through $(x, t)$. Note that the time coordinate $t_{0}$ of this point must lie in the interval $\left[0, t_{c}\right]$. We have

$$
x-a\left(t_{0}\right)=\Lambda\left(t_{0}\right)\left(t-t_{0}\right),
$$

where

$$
\Lambda\left(t_{0}\right)=\lambda_{+}\left(a\left(t_{0}\right), t_{0}\right)=V\left(a\left(t_{0}\right), t_{0}\right)+\sqrt{N\left(a\left(t_{0}\right), t_{0}\right)}=\sqrt{Q}-\frac{3}{2} \beta_{0} t_{0},
$$

since $V\left(a\left(t_{0}\right), t_{0}\right)=-\beta_{0} t_{0}$ and $N\left(a\left(t_{0}\right), t_{0}\right)=\left(\sqrt{Q}-\beta_{0} t_{0} / 2\right)^{2}$. Since $R_{+}$is constant along (3.22) and $R_{-}$is constant on the whole region $S$, we have

$$
V(x, t)=V\left(a\left(t_{0}\right), t_{0}\right), \quad N(x, t)=N\left(a\left(t_{0}\right), t_{0}\right),
$$




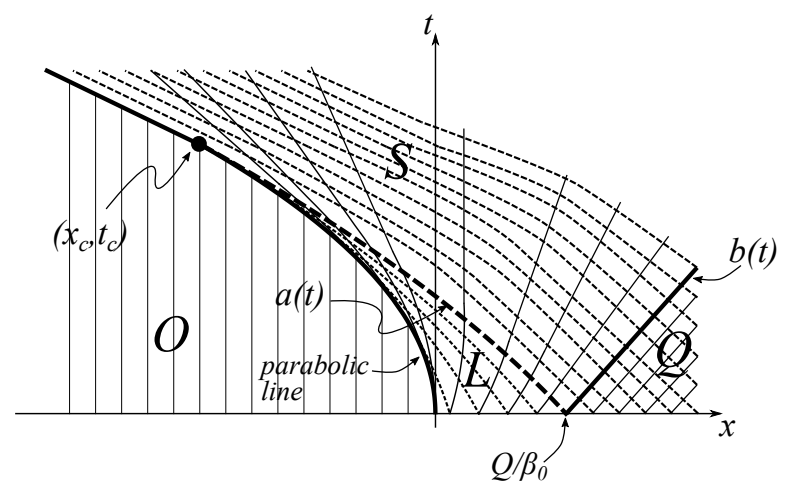

FIG. 4. Positive (continuous line) and negative (dashed line) characteristics corresponding to the initial data $u(x, 0)=0$ and (3.10). The coordinates of the point $C$ are $x_{c}=-2 Q / \beta_{0}$ and $t_{c}=2 \sqrt{Q} / \beta_{0}$. There are four different regions: on the left, the region $O$ where the elevation $\eta$ vanishes; on the right, the region $Q$ where $\eta=Q$; the interface is a straight line in $L$, and is given by the simple wave (3.26) in $S$.

and we are left with solving (3.22) with respect to $t_{0}$. Thanks to (3.23), this is a quadratic equation, and from Figure 4 it is clear that we are interested in the smaller of the two real solutions; hence

$$
t_{0}=\frac{1}{5 \beta_{0}}\left(4 \sqrt{Q}+3 t \beta_{0}-\sqrt{20 \beta_{0} x-4 Q+4 t \beta_{0} \sqrt{Q}+9 t^{2} \beta_{0}^{2}}\right) .
$$

This yields the explicit form of $V$, and so $N$, by using the simple wave relation from the "-" Riemann invariant $R_{-}=V-2 \sqrt{N}=-2 \sqrt{Q}$,

$$
\begin{aligned}
& V(x, t)=\frac{1}{5}\left(\sqrt{20 \beta_{0} x-4 Q+4 t \beta_{0} \sqrt{Q}+9 t^{2} \beta_{0}^{2}}-4 \sqrt{Q}-3 t \beta_{0}\right), \\
& N(x, t)=\frac{1}{4}(V(x, t)+2 \sqrt{Q})^{2} .
\end{aligned}
$$

A useful observation for the qualitative characterization of this solution is the presence of the fixed point $x^{*}=2 Q /\left(9 \beta_{0}\right)$ where the interface height remains constant at all times,

$$
N\left(\frac{2 Q}{9 \beta_{0}}, t\right)=\frac{4 Q}{9} .
$$

Note that this height is the same as that for the classical dam-break of section 2, where, however, the special point at which the value $\eta=4 Q / 9$ is attained remains fixed at the origin $x^{*}=0$.

The straight-line part of the solution shrinks to the point $x_{c}=-2 Q / \beta_{0}$ when $t=t_{c}=2 \sqrt{Q} / \beta_{0}$. Note that at this time the shoulder, simple wave component of the solution becomes a straight line with slope $\beta_{0} / 5$, or

$$
N\left(x, t_{c}\right)=\frac{\beta_{0}}{5} x+\frac{2 Q}{5}, \quad p\left(t_{c}\right)=-\frac{2 Q}{\beta_{0}} \leq x \leq \frac{3 Q}{\beta_{0}}=b\left(t_{c}\right),
$$

and the concavity of the shoulder switches from negative to positive at this time. After $t=t_{c}$, the contact point with the bottom moves at constant speed $-2 \sqrt{Q}$, $x=p(t)=2 Q / \beta_{0}-2 t \sqrt{Q}$, and the solution becomes 


$$
\eta(x, t)=\left\{\begin{array}{ll}
0, & x<p(t), \\
N(x, t), & p(t) \leq x \leq b(t), \\
Q, & x>b(t),
\end{array} \quad u(x, t)= \begin{cases}0, & x<p(t), \\
V(x, t), & p(t) \leq x \leq b(t), \\
0, & x>b(t) .\end{cases}\right.
$$

Thus, for times $t>t_{c}$, in the region $[p(t), b(t)]$ the solution is a simple wave, just as the Stoker rarefaction wave of section 2. This evolution is sketched in Figure 5. However, as remarked above, the jump discontinuity in the velocity field at the point where $\eta$ vanishes cannot be eliminated at all times $t>0$ by a choice of $u$ consistent with the Hopf equation to which system (1.1) reduces for $\eta=0$. This is essentially due to the time evolution of $u$ in the linear region $(p(t), a(t))$ where $u(x, t)=-\beta_{0} t$, which would require a time dependent continuation of $u$ for $x<p(t)$.

Suppose in fact that for the region $\eta=0$ we were to look for a solution of the initial-boundary value problem for the second equation of system (1.1), i.e., the Hopf equation

$$
u_{t}+u u_{x}=0, \quad u(x, 0)=f(x), \quad u(p(t), t)=-\beta_{0} t \quad \text { for } x<p(t),
$$

for some function $f(x)$. Then the change of variables $x=y+p(t)$ and $u(x, t)=$ $v(y, t)-\beta_{0} t$ transforms this into the equivalent initial-boundary value problem of the fixed half-line domain,

$$
v_{t}+v v_{y}=\beta_{0}, \quad v(y, 0)=g(y), \quad v(0, t)=0, \quad \text { for } y<0,
$$

whose solution, by characteristics, i.e.,

$$
\frac{d v}{d t}=\beta_{0} \quad \text { on curves } y(t) \text { such that } \quad \frac{d y}{d t}=v(y(t), t)
$$

is

$$
v(y, t)=g\left(y_{0}\right)+\beta_{0} t, \quad y=y_{0}+g\left(y_{0}\right) t+\frac{1}{2} \beta_{0} t^{2}
$$

with $g$ a function such that $g(0)=0$ to be compatible with the boundary condition at $t=0$, and otherwise must be extended to maintain the boundary condition $v(0, t)=0$. This implies that a characteristic emanating from $y=y_{0}$ at time $t=0$ must be such that

$$
g\left(y_{0}\right)=-\beta_{0} t
$$

at time $t$, so that the corresponding characteristic point of origin $y_{0}$ is given by

$$
0=y_{0}-\frac{1}{2} \beta_{0} t^{2}
$$

i.e., only characteristics emanating from the positive semiaxis $y>0$ can be considered. Thus, the choice of the function $g$ for the support $y_{0} \geq 0$ is $g\left(y_{0}\right)=-\sqrt{2 \beta_{0} y_{0}}$, so that that these characteristics are part of the family of parabolae

$$
y=y_{0}-\sqrt{2 \beta_{0} y_{0}} t+\frac{1}{2} \beta_{0} t^{2},
$$

whose vertex is tangent to the $t$-axis. The latter is, in fact, the envelope of the characteristics family as shown by the second equation in (3.32); by this and the 
boundary condition $v(y(t), t)=0$ it can be checked that the envelope is itself a characteristic, which of course corresponds to the "parabolic" limit $v=0$ for (3.31). The corresponding solution $v(y, t)$ on the half line $y>0$ can be taken to be time independent,

$$
v(y, t)=-\sqrt{2 \beta_{0} y}
$$

as can be readily checked. No characteristics emanating from $y_{0}>0$ can be prolonged into the negative half-plane $y<0$. Together with the fact that regardless of the choice of initial data $g\left(y_{0}\right)$ all characteristic curves eventually grow as $\beta_{0} t^{2} / 2$ at long times, this shows that the boundary condition at $y=0$ cannot be met by regular solutions of (3.32) for $y_{0}<0$. Thus, the construction of solutions of the initial value problem (3.10) for system (1.1) in the dry (vacuum) region cannot be carried out without some form of shock fitting for the velocity component. The choice $u=0$ for $x<p(t)$ is the simplest one that satisfies the shock velocity jump condition (2.7), whence the conservation laws discussed in section 2 follow for this case of piecewise linear initial data as well.

However, an alternative definition of the velocity $u$ in the vacuum region, akin to that used for the Riemann problem to extend this variable continuously without using a shock, can also be made in this case. By using the pair $\eta, m$ of dependent variables instead of $\eta, u$, where $m$ is the momentum of the fluid, the Airy system can be written as

$$
\eta_{t}+m_{x}=0, \quad m_{t}+\frac{2 m}{\eta} m_{x}-\frac{m^{2}}{\eta^{2}} \eta_{x}+\eta \eta_{x}=0,
$$

and the velocity $u$ can be defined by the ratio $m / \eta$. Of course, in the region where $\eta$ vanishes, this definition loses meaning, but the velocity could still be extended by using the appropriate limit $\eta \rightarrow 0$ in such a way that the ratio $m / \eta$ stays bounded. For the linear core case under consideration in this section, it is natural to continue $u(x, t)$ with the value of this limit as $x \rightarrow p(t)^{-}$, that is, assume

$$
u(x, t)=-\beta_{0} t, \quad x \leq p(t)
$$

for times $t<t_{c}$ and

$$
u(x, t)=-2 \sqrt{Q}, \quad x \leq-2 \sqrt{Q} t
$$

for times $t>t_{c}$, i.e., after the linear core disappears, in analogy with the Stoker rarefaction wave case. This alternative formulation of the evolution would effectively turn the initial value problem with piecewise data (3.10) on the infinite line into an initial-boundary value problem on the half line $x>p(t)$, with Cauchy data on the velocity $u$ at the moving boundary $x=p(t)$.

4. Dam-break: the parabolic connection case. In this section we consider the evolution from a dry region connected by a parabolic profile to a constant height $Q$. We begin with the simplest situation of a "global" simple wave (see, e.g., [9]), leading to an initial condition obtained by a backward-time extension of the Riemann problem, which yields a closed form and explicit expression illustrating the effect of $C^{1}$ connections with the dry case. The price to pay for simplicity is that of nonvanishing velocity boundary conditions at infinity in the fluid region. Next, we turn to a class of initial conditions which can still result in exact solutions, albeit implicitly defined. Just as in the previous linear case, splicing together closed form solutions results into overall $C$-functions which are nondifferentiable at the connection point with a background quiescent state of thickness $Q$, with vanishing velocity boundary conditions at infinity. 

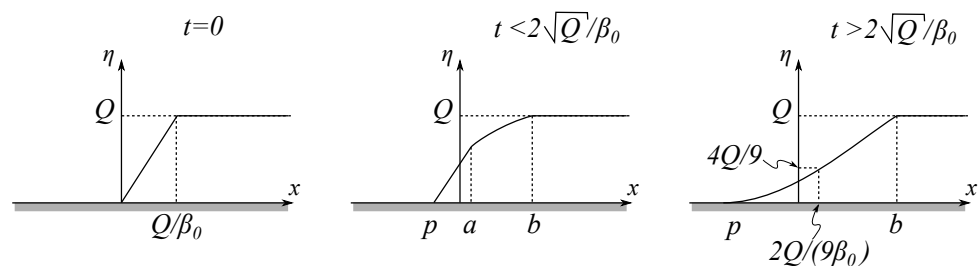

FIG. 5. Sketch of the evolution of $\eta(x, t)$ starting from the initial data (3.10). The relevant $x$-values are $p(t)=-\beta_{0} t^{2} / 2$ for $0 \leq t \leq t_{c} \equiv 2 \sqrt{Q} / \beta_{0}, p(t)=2 Q / \beta_{0}-2 t \sqrt{Q}$ for $t>t_{c}$, and $a(t)=Q / \beta_{0}-t \sqrt{Q}-\beta_{0} t^{2} / 4, b(t)=Q / \beta_{0}+t \sqrt{Q}$.

4.1. Simple wave setup. Consider the initial conditions for the shallow water (Airy) model defined by

$$
\begin{gathered}
\eta(x, 0)=\left\{\begin{array}{cl}
0, & x<0, \\
x^{2} /\left(9 t_{c}^{2}\right), & 0<x<3 \sqrt{Q} t_{c}, \\
Q, & x>3 \sqrt{Q} t_{c},
\end{array}\right. \\
u(x, 0)=\left\{\begin{array}{cl}
0, & x<0, \\
-2 x /\left(3 t_{c}\right), & 0<x<3 \sqrt{Q} t_{c}, \\
-2 \sqrt{Q}, & x>3 \sqrt{Q} t_{c} .
\end{array}\right.
\end{gathered}
$$

For $t<t_{c}$ this initial conditions evolve as

$$
\eta(x, t)=\left\{\begin{array}{cl}
0, & x<0, \\
\frac{1}{9}\left(\frac{x}{t_{c}-t}\right)^{2}, & 0<x<3 \sqrt{Q}\left(t_{c}-t\right), \\
Q, & x>3 \sqrt{Q}\left(t_{c}-t\right),
\end{array}\right.
$$

$$
u(x, t)=\left\{\begin{array}{cl}
0, & x<0, \\
-\frac{2}{3} \frac{x}{t_{c}-t}, & 0<x<3 \sqrt{Q}\left(t_{c}-t\right), \\
-2 \sqrt{Q}, & x>3 \sqrt{Q}\left(t_{c}-t\right) .
\end{array}\right.
$$

This solution is a simple wave everywhere for $t<t_{c}$ of class $C^{1}$ at $x=0$ and $C$ at $x=3 \sqrt{Q} t_{c}$. The constant Riemann invariant is

$$
u+2 \sqrt{\eta}=0 .
$$

The dry point at which $\eta=0$ is fixed at $x=0$ until the gradient catastrophe time $t=t_{c}$. At this time the solution becomes the pair of Heaviside functions

$$
\eta\left(x, t_{c}\right)=\left\{\begin{array}{ll}
0, & x<0, \\
Q, & x>0,
\end{array} \quad u\left(x, t_{c}\right)=\left\{\begin{array}{cc}
0, & x<0 \\
-2 \sqrt{Q}, & x>0
\end{array}\right.\right.
$$



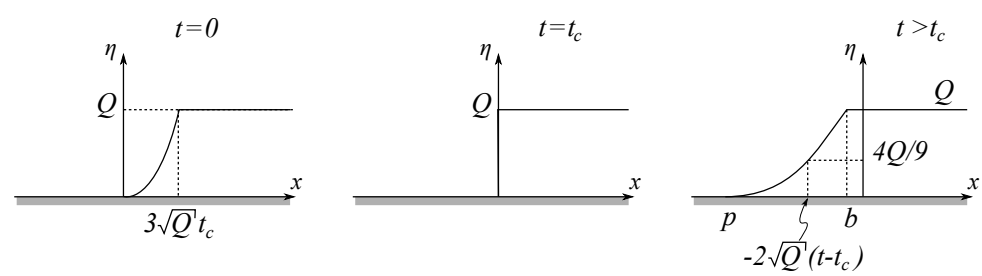

FIG. 6. Sketch of the evolution of $\eta(x, t)$ starting from the initial data (4.1).

A suitable regularization has to be adopted in order to continue the solution after the catastrophe at $t=t_{c}$. Simply continuing the solution (4.2) for $t>t_{c}$ forces a shock to be fitted for the velocity $u$, which would then connect a positive value to the negative right asymptotic value $-2 \sqrt{Q}$. This would violate the shock conditions, since at the shock position $\eta$ is continuous and positive. Another option, which would preserve the simple wave structure, would be to choose a different Riemann invariant to be kept constant overall. As shown by (4.3), the early time solution is a simple wave with the constant Riemann invariant $u+2 \sqrt{\eta}=0$. After $t_{c}$ the only possible choice is to fix, using the nonzero asymptotic values of the fields, the other Riemann invariant to be

$$
u-2 \sqrt{\eta}=-4 \sqrt{Q} .
$$

The related solution is then

$$
\begin{gathered}
\eta(x, t)=\left\{\begin{array}{cl}
0, & x<p(t), \\
\frac{1}{9}\left(\frac{x}{t-t_{c}}+4 \sqrt{Q}\right)^{2}, & p(t)<x<b(t), \\
Q, & x>b(t),
\end{array}\right. \\
u(x, t)=\left\{\begin{array}{cl}
0, & x<p(t), \\
\frac{2}{3}\left(\frac{x}{t-t_{c}}-2 \sqrt{Q}\right), & p(t)<x<b(t), \\
-2 \sqrt{Q}, & x>b(t) .
\end{array}\right.
\end{gathered}
$$

where $b(t)=-\sqrt{Q}\left(t-t_{c}\right)$ and $p(t)=-4 \sqrt{Q}\left(t-t_{c}\right)$. This solution is a simple wave preserving (4.5) only in the regions where the fields are nonzero; therefore one has to check that splicing the two parts (4.2) and (4.6) into a continuous solution for $\eta$ is compatible with shock and gluing conditions. At $x=p(t)=-4 \sqrt{Q}\left(t-t_{c}\right)$, the rightmost point where $\eta$ is zero, the velocity $u$ has a shock which is admissible because the shock speed is equal to the value of the velocity $u$ to the right of this point. The evolution of the solution is a sort of "stumble" at time $t=t_{c}$ caused by the persistence of the dry point at $x=0$, followed by a "slide" as the Riemanm dam-break after $t=t_{c}$. This evolution is sketched in Figure 6 .

Remark 4.1. The Airy system is invariant under Galileian boosts. This property is reflected by the fact that, in the region where $\eta$ is nonzero, the solution (4.6) coincides, up to a change of inertial reference frame, with that of the classical zero initial velocity Riemann problem of section 2; that is, in this region (4.6) is obtained from (2.6) by the boost

$$
u=u_{R}-2 \sqrt{Q}, \quad \eta=\eta_{R}, \quad x=x_{R}+2 \sqrt{Q}\left(t-t_{c}\right), \quad t=t_{R}+t_{c},
$$


where the subscript $R$ indicates solution (2.6). This can be also further verified observing that the point where $\eta=4 Q / 9$ is moving to a constant velocity,

$$
x=-2 \sqrt{Q}\left(t-t_{c}\right) .
$$

The fluid thicknesses $\eta=0, \eta=4 Q / 9$, and $\eta=Q$ are the only ones whose velocities are constant in time.

4.2. Parabolic core exact solution. When a quadratic polynomial expression is sought for $\eta(x, t)$ in system (1.1), self-similar parabolic solutions (already briefly mentioned in [17]) can be determined of the form

$$
u(x, t)=\nu(t) x, \quad \eta(x, t)=\gamma(t) x^{2},
$$

where the coefficients satisfy the system of ODE

$$
\dot{\nu}+\nu^{2}+2 \gamma=0, \quad \dot{\gamma}+3 \nu \gamma=0 .
$$

If we consider the initial conditions $\nu(0)=0$ and $\gamma(0)=\gamma_{0}>0$, then it can be checked that the curvature $\gamma$ satisfies the ODE

$$
\dot{\gamma}^{2}=36\left(\gamma^{3}-\gamma_{0}^{1 / 3} \gamma^{8 / 3}\right)
$$

and is an increasing function of time. After introducing the auxiliary variable $\sigma=$ $\left(\gamma / \gamma_{0}\right)^{1 / 3}$, the previous equation simplifies to

$$
\dot{\sigma}=2 \sqrt{\gamma_{0}} \sigma^{2} \sqrt{\sigma-1}
$$

and we have that

$$
\nu^{2}=4 \gamma_{0}\left(\sigma^{3}-\sigma^{2}\right), \quad \gamma=\gamma_{0} \sigma^{3},
$$

where $\sigma(t)$ is the inverse of

$$
t(\sigma)=\frac{\sqrt{\sigma-1}+\sigma \arctan (\sqrt{\sigma-1})}{2 \sqrt{\gamma_{0}} \sigma}
$$

and satisfies the initial condition $\sigma(0)=1$. The characteristics are given by

$$
x_{ \pm}(\sigma)=x_{0} \frac{\sqrt{\sigma} \pm \sqrt{\sigma-1}}{\sigma} .
$$

This and other self-similar solutions in the quadratic class have been studied in more detail in [3] and [2].

4.3. Piecewise parabolic-constant solutions. We now consider initial conditions obtained by splicing together the parabolic core above with a quiescent constant background state, as done for the linear core case of section 3.2. Specifically, we take $u(x, 0)=0$ and

$$
\eta(x, 0)= \begin{cases}0, & x<0 \\ \gamma_{0} x^{2}, & 0 \leq x \leq a_{0} \\ Q, & x>a_{0},\end{cases}
$$


where $\gamma_{0}, Q>0$ and $a_{0}=\sqrt{Q / \gamma_{0}}$. For $x \geq 0$, the evolution is the same obtained by taking $\eta(x, 0)$ even, coinciding with (4.16). Indeed, this case has been described in $[3,2]$, where we have focused on the consequences that $\eta(0, t)=0$ for all $t>0$. (This is a general fact, related to the initial data being $C^{1}$ in a neighborhood of $x=0$.) Hence the solution we are looking for can be obtained by gluing together $(\eta(x, t), u(x, t))=(0,0)$ for $x<0$ with the solution above for $x \geq 0$, before a certain catastrophe time $t_{c}$.

For initial data (4.16), there is only one nondifferentiable point, $x=a_{0}$, and we know (see section 3.2 and $[2,3]$ ) that it splits and evolves along the negative characteristic

$$
x=a(\sigma)=\sqrt{\frac{Q}{\gamma_{0}}} \frac{\sqrt{\sigma}-\sqrt{\sigma-1}}{\sigma},
$$

where $\sigma(t)$ is the inverse of (4.14), and the positive characteristic (associated with the constant solution $\eta=Q, u=0)$,

$$
x=b(t)=a_{0}+t \sqrt{Q} .
$$

The behavior of $(\eta(x, t), u(x, t))$ for as long as continuity is maintained by the evolution is given by

$$
\eta(x, t)=\left\{\begin{array}{ll}
0, & x \leq 0, \\
\gamma(t) x^{2}, & 0<x<a(t), \\
N(x, t), & a(t) \leq x \leq b(t), \\
Q, & x>b(t),
\end{array} \quad u(x, t)= \begin{cases}0, & x \leq 0, \\
\nu(t) x, & 0<x<a(t), \\
V(x, t), & a(t) \leq x \leq b(t), \\
0, & x>b(t),\end{cases}\right.
$$

where $\nu(t)$ and $\gamma(t)$ are defined by the solutions of the ODEs above, and the pair $(N, V)$ is a simple wave solution of system (1.1) in the time-varying interval $[a(t), b(t)]$, such that the (negative sign) Riemann invariant is $V-2 \sqrt{N}=-2 \sqrt{Q}$. This simple wave solution is

$$
N(x, t)=\gamma\left(\sigma_{0}\right) a\left(\sigma_{0}\right)^{2}=\sigma_{0} Q\left(\sqrt{\sigma_{0}}-\sqrt{\sigma_{0}-1}\right)^{2},
$$

the function $\sigma_{0}=\sigma_{0}(x, t)$ being implicitly defined by

$$
x=\Lambda\left(\sigma_{0}\right)\left(t-\frac{\sqrt{\sigma_{0}-1}+\sigma_{0} \arctan \left(\sqrt{\sigma_{0}-1}\right)}{2 \sigma_{0} \sqrt{\gamma_{0}}}\right)+\frac{\sqrt{Q \sigma_{0}}-\sqrt{Q\left(\sigma_{0}-1\right)}}{\sigma_{0} \sqrt{\gamma_{0}}},
$$

where

$$
\Lambda\left(\sigma_{0}\right)=3 \sqrt{Q} \sigma_{0}\left(1-\sqrt{1-\frac{1}{\sigma_{0}}}-\frac{2}{3 \sigma_{0}}\right) .
$$

The interface described by (4.20) admits an invariant thickness value, similar to what was observed for the solutions in the previous sections. Indeed, inverting (4.20) one finds

$$
\sigma_{0}=\frac{N}{\sqrt{Q}(2 \sqrt{N}-\sqrt{Q})}
$$



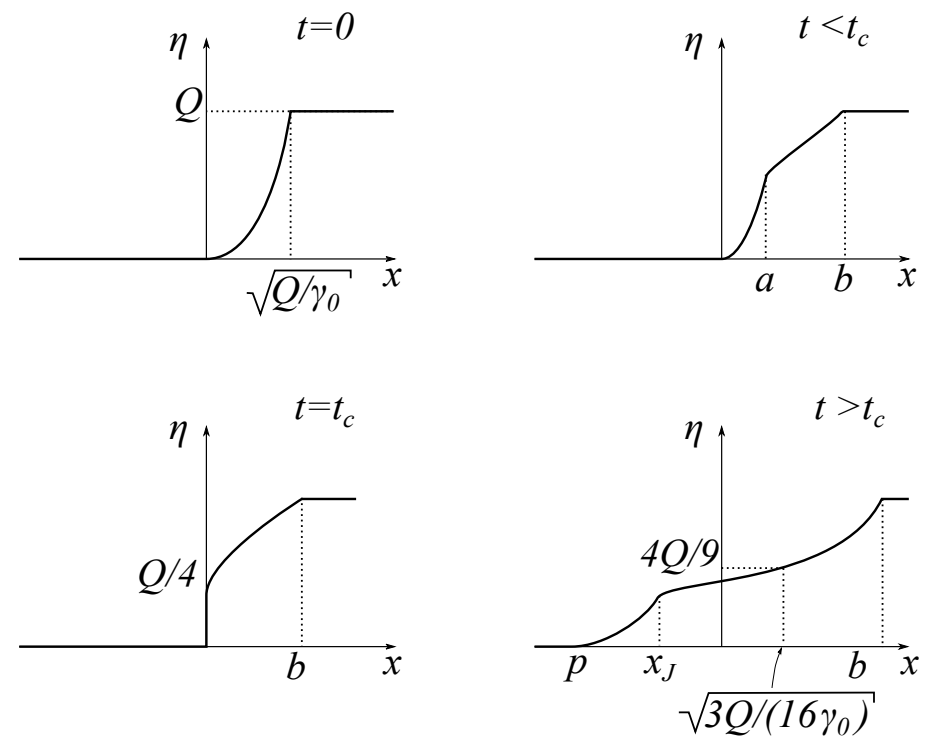

FIG. 7. Sketch of the evolution of $\eta(x, t)$ starting from the initial data (4.16) and $u(x, 0)=$ 0 . The relevant $x$-values are $x=a(t)$ (see (4.17) and (4.14)), $b(t)=t \sqrt{Q}+\sqrt{Q / \gamma_{0}}, p(t)=$ $-2 \sqrt{Q}\left(t-\pi /\left(4 \sqrt{\gamma_{0}}\right)\right)$, and $x_{J}(t)=-\sqrt{Q}\left(t-\pi /\left(4 \sqrt{\gamma_{0}}\right)\right) / 2$.

and substituting in (4.21), one obtains an implicit expression for $N(x, t)$. Using this, it is easy to show that

$$
N\left(\sqrt{\frac{3 Q}{16 \gamma_{0}}}, t\right)=\frac{4 Q}{9} .
$$

The coalescence time (i.e., the time when $a(t)$ becomes zero, so that the parabola collapses to a vertical segment) is

$$
t_{c}=\frac{\pi}{4 \sqrt{\gamma_{0}}},
$$

which is the global gradient catastrophe time mentioned above (see [2] for the details and [3] for more information on the relevant features of this construction).

As seen for the Riemann construction of section 4.1, after this gradient catastrophe the solution can be continued by considering another simple wave such that its corresponding Riemann invariant is $V_{0}-2 \sqrt{N_{0}}=-2 \sqrt{Q}$. Thus, define the new pair of dependent variables $\left(N_{0}, V_{0}\right)$

$$
N_{0}=\frac{1}{9}\left(\frac{x}{t-t_{c}}+2 \sqrt{Q}\right)^{2}, \quad V_{0}=\frac{2}{3}\left(\frac{x}{t-t_{c}}-\sqrt{Q}\right),
$$

and, as seen from the value of the Riemann invariant $R_{-}$, the pairs $(N, V)$ and $\left(N_{0}, V_{0}\right)$ form a unique simple wave. The gluing point between them can be determined as follows. Denote the leftmost point of $N$ by $x_{J}(t)$, which corresponds to $\sigma_{0} \rightarrow+\infty$ in (4.21) and (4.20). We obtain

$$
x_{J}(t)=-\frac{\sqrt{Q}}{2}\left(t-\frac{\pi}{4 \sqrt{\gamma_{0}}}\right), \quad N\left(x_{J}(t), t\right)=\frac{Q}{4}, \quad V\left(x_{J}(t), t\right)=-\sqrt{Q} .
$$




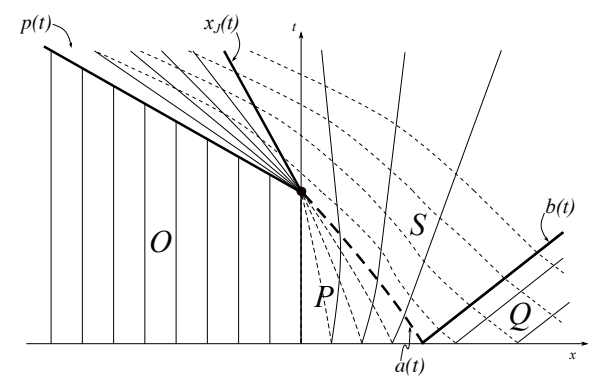

FIG. 8. Positive (continuous line) and negative (dashed line) characteristics corresponding to the initial data $u(x, 0)=0$ and (4.16). There are four different regions: on the left, the region $O$ where the elevation $\eta$ vanishes; on the right, the region $Q$ where $\eta=Q$; the interface is a parabola in $P$, and is given by the simple waves $(N, V)$ and $\left(N_{0}, V_{0}\right)$ in $S$.

It can be seen immediately that the rarefaction wave (4.26) passes through this point, since

$$
N_{0}\left(x_{J}(t), t\right)=\frac{Q}{4},
$$

and hence at $x_{J}(t)$ the simple waves $N_{0}$ and $N$, respectively, for $x<x_{J}(t)$ and $x>x_{J}(t)$, form a continuous function. Figure 7 provides a sketch of the evolution of the solution, while Figure 8 illustrates its interpretation by characteristics in the $(x, t)$ plane.

Remark 4.2. The $x$-derivative of the solution is also continuous at $x_{J}(t)$, since

$$
N_{0 x}\left(x_{J}(t), t\right)=N_{x}\left(x_{J}(t), t\right)=\frac{\sqrt{Q}}{3\left(t-t_{c}\right)},
$$

where $N_{x}\left(x_{J}(t), t\right)$ is computed as

$$
N_{x}\left(x_{J}(t), t\right)=\lim _{\sigma_{0} \rightarrow+\infty} \frac{\partial N\left(x\left(\sigma_{0}, t\right), t\right) / \partial \sigma_{0}}{\partial x\left(\sigma_{0}, t\right) / \partial \sigma_{0}},
$$

using again (4.21) and (4.20). The simple wave definition then assures that the same continuity properties hold for the velocity field components $V_{0}$ and $V$. However, note that by a similar argument the one-sided second derivative $N_{x x}(x, t)$ can be computed and diverges, $N_{x x} \rightarrow-\infty$ as $x \rightarrow x_{J}(t)$, for all times $t>t_{c}$ (details omitted here).

5. Asymptotic behaviour of rarefaction waves: "unfolding variables". For all the dam-break scenarios studied so far, the long time evolution resembles that of rarefaction simple waves. So for instance, in Figure 9 we plot the evolution of the speed of the foot of the interface for different classes of initial data, i.e., Riemann-like, linear, and parabolic. In the last two cases we choose $\beta_{0}$ and $\gamma_{0}$ in order to have the same support $x \in\left[0, a_{0}\right]$ the nonconstant part of the initial data,

$$
\beta_{0}=\frac{Q}{a_{0}}, \quad \gamma_{0}=\frac{Q}{\left(a_{0}\right)^{2}} .
$$

Memory of differences in the initial conditions are, however, kept for all times in the evolution, and a precise statement about the long time approach to a simple wave for the transition between the dry region and the quiescent state of constant thickness fluid layer seems desirable. For a single nonlinear conservation law, the 

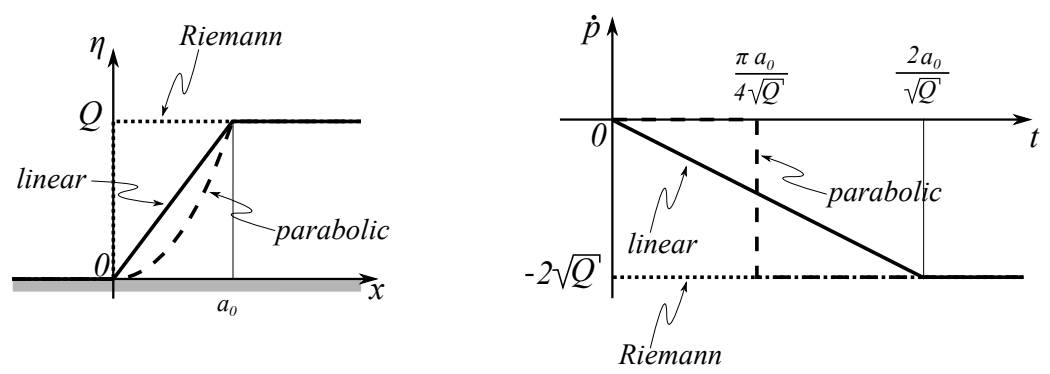

FIG. 9. Contact dry point $p(t)$ evolution. Left panel: sketch of the $\eta$ initial data for the Riemann (short dash), linear core (long dash), and parabolic core (solid) profiles; right panel: velocity $\dot{p}$ for the different classes of initial data, labelled accordingly.

mean of the initial data is an invariant in time (as is easy to observe for strong solutions and confirmed by a classical theorem [13] for weak ones). All solutions of the previous sections at some finite time become piecewise continuous combinations of simple waves, and as such they are solutions of a single conservation law, so that the mean must be conserved. Thus, for all cases we have considered, differences that may persist at long times must be compatible with the fact that the same mean is maintained at these times, an invariant that is essentially set by the quiescent state thickness $Q$. In all cases, note that memory of the initial conditions at all times is kept by time independent shifts which cannot be canceled by a Galileian boost. Two of these differences immediately appear in the positions of the nondifferentiable points in the graph of $\eta$. Thus, for instance, for the linear case even if both points corresponding to $\eta=0$ and $\eta=Q$ have the same asymptotic velocity in time, the one at $\eta=Q$ is shifted by $Q / \beta_{0}$, while the "foot" at $\eta=0$ is shifted by $2 Q / \beta_{0}$. Moreover, another shift applies to the unique point $x^{*}$ where the thickness $\eta$ matches the invariant $\eta\left(x^{*}, t\right)=4 Q / 9$. In the classical dam-break case, we have $x^{*}=0$, while in the linear case $x^{*}=2 Q /\left(9 \beta_{0}\right)$. Of course, these shifts are all compatible with maintaining the mean value of the solution constant.

In order to isolate differences in how the long time asymptotics of initial data varies around the mean it is useful to adapt an idea developed for shocks in [2], which relies on the use the stretched variables (see, e.g., [12]). For the classical Riemann dam-break of section 2, the interval $-2 \sqrt{Q} t<x<\sqrt{Q} t$ where the solution (2.6) varies in space and time can be fixed by rescaling the variable $x$ as

$$
\xi=\frac{x}{3 \sqrt{Q} t}
$$

with $-2 / 3<\xi<1 / 3$. In these variables the solution, being self-similar in $x / t$, does not depend on time, and it turns into a simple polynomial of the variable $\xi$,

$$
\eta=\frac{Q}{9}(3 \xi+2)^{2}, \quad u=\frac{2 \sqrt{Q}}{3}(3 \xi-1) .
$$

A similar approach can be used for the study of the exact solutions in sections 3.2 and 4 for large times $t>2 \sqrt{Q} / \beta_{0}$, when the polynomial "core" has disappeared, either by being absorbed by the shoulder simple wave, as for the linear case, or by the gradient catastrophe collapse, as in the parabolic case, respectively.

We study the linear case first. The $(x, t)$ varying part of the linear dam-break solution between $2 Q / \beta_{0}-2 t \sqrt{Q}<x<Q / \beta_{0}+t \sqrt{Q}$ can be written in the new "unfolding" variable 


$$
\xi_{l}=\left(x-\frac{4 Q}{3 \beta_{0}}\right) /\left(3 \sqrt{Q} t-\frac{Q}{\beta_{0}}\right)
$$

whose range is fixed between $-2 / 3$ and $1 / 3$. The time asymptotic behavior of the fields in the new variable is

$$
N\left(x\left(\xi_{l}, t\right), t\right)=\frac{Q}{9}\left(3 \xi_{l}+2\right)^{2}+O\left(t^{-1}\right), \quad V\left(x\left(\xi_{l}, t\right), t\right)=\frac{2 \sqrt{Q}}{3}\left(3 \xi_{l}-1\right)+O\left(t^{-1}\right)
$$

as $t \rightarrow+\infty$. Thus, apart from the spatial shifts discussed above, the solution pair $(N, V)$ has the same asymptotic behaviour as that of the Riemann dam-break in the unfolding variables, with the remainder, in these variables, decaying as $O\left(t^{-1}\right)$. Note that the limit $\beta_{0} \rightarrow+\infty$ correctly reproduces the classical Riemann dam-break.

Remark 5.1. Note that a direct approach to the long time limit at $x$ fixed of the nonconstant part (3.26) of the linear dam-break solution,

$$
V(x, t)=\frac{2}{3}\left(\frac{\tilde{x}}{t}-\sqrt{Q}\right)-\frac{4 \sqrt{Q}}{27 \beta_{0}} \frac{\tilde{x}}{t^{2}}+O\left(t^{-3}\right), \quad \tilde{x}=x-\frac{2 Q}{9 \beta_{0}},
$$

yields artificial corrections for the interface variable asymptotics. In fact, the lowest terms of the asymptotic velocity are the same as in the classical dam-break case (2.6), the only difference being the presence of $\tilde{x}$ instead of $x$; the translation $x \mapsto \tilde{x}$ corresponds to setting the invariant interface height at $x=0$. However, the asymptotic series for the interface is

$$
N(x, t)=\frac{1}{9}\left(\frac{\tilde{x}}{t}+2 \sqrt{Q}\right)^{2}-\frac{8 Q}{81 \beta_{0}} \frac{\tilde{x}}{t^{2}}+O\left(t^{-3}\right),
$$

which leads to a different $O\left(t^{-2}\right)$ behavior with respect to the expression for Riemann dam-break rarefaction wave, the first term in parenthesis.

The case of the initial parabolic core can be treated similarly. The appropriate unfolding variable is now

$$
\xi_{p}=\left(x-\frac{\pi+4}{6} \sqrt{\frac{Q}{\gamma_{0}}}\right) /\left(3 \sqrt{Q} t-\frac{\pi-2}{2} \sqrt{\frac{Q}{\gamma_{0}}}\right)
$$

where the denominator is the postshock length of the $(x, t)$-varying part of the parabolic dam-break and the $x$-shift is again chosen in such a way that the domain of the unfolding variable $\xi_{p}$ is between $-2 / 3$ and $1 / 3$. The unfolded equivalent to the point $x_{J}(t)$ is

$$
\xi_{p_{J}}=\frac{\pi+16+12 \sqrt{\gamma_{0}} t}{12(\pi-2)-72 \sqrt{\gamma_{0}} t}
$$

This point is not fixed in time, but it reaches an asymptotic value

$$
\xi_{p_{J}} \rightarrow-\frac{1}{6} \quad \text { for } \quad t \rightarrow+\infty
$$


TABLE 1

Comparison of self-similar intermediate behavior of different dam-break configurations of increasing degree of regularity. Here $\chi_{\beta}(x)$ and $\chi_{\gamma}(x)$ denote the characteristic functions of the intervals $\left(0, Q / \beta_{0}\right)$ and $\left(0, \sqrt{Q} / \gamma_{0}\right)$, respectively, and $H$ is the Heaviside function.

\begin{tabular}{|c|c|c|c|c|}
\hline $\begin{array}{c}\text { Initial interface } \eta(x, 0) \\
(u(x, 0)=0)\end{array}$ & $\begin{array}{c}\text { unfolding variable } \\
\frac{x-q}{3 \sqrt{Q} t-l}\end{array}$ & $\begin{array}{c}\text { foot } p(t) \\
-2 \sqrt{Q} t+k\end{array}$ & $\begin{array}{c}\text { junction } b(t) \\
\sqrt{Q} t+m\end{array}$ & $\begin{array}{c}\text { height } \\
\eta\left(x_{*}, t\right)=\frac{4}{9} Q\end{array}$ \\
\hline$Q H(x)$ & $q=0, l=0$ & $k=0$ & $m=0$ & $x_{*}=0$ \\
\hline$\beta_{0} x \chi_{\beta}(x)+Q H\left(x-\frac{Q}{\beta_{0}}\right)$ & $q=\frac{4 Q}{3 \beta_{0}}$ & $k=\frac{2 Q}{\beta_{0}}$ & $m=\frac{Q}{\beta_{0}}$ & $x_{*}=\frac{2 Q}{9 \beta_{0}}$ \\
\hline $\begin{array}{l}l=-\frac{Q}{\beta_{0}} \\
\gamma_{0} x^{2} \chi_{\gamma}(x)+Q H\left(x-\sqrt{\frac{Q}{\gamma_{0}}}\right)\end{array}$ & $q=\frac{\pi+4}{6} \sqrt{\frac{Q}{\gamma_{0}}}$ & $k=\frac{\pi}{2} \sqrt{\frac{Q}{\gamma_{0}}}$ & $m=\sqrt{\frac{Q}{\gamma_{0}}}$ & $x_{*}=\sqrt{\frac{3 Q}{16 \gamma_{0}}}$ \\
\hline
\end{tabular}

which is the midpoint in the interval of the variable $\xi_{p}$. Using the implicit solution (4.23) shows, once again, that the asymptotic behavior of the combined $(N, V)$ and $\left(N_{0}, V_{0}\right)$ pair, $(\widetilde{N}, \widetilde{V})$, say, is

$$
\begin{aligned}
& \widetilde{N}\left(x\left(\xi_{p}, t\right), t\right)=\frac{Q}{9}\left(3 \xi_{p}+2\right)^{2}+O\left(t^{-1}\right), \\
& \widetilde{V}\left(x\left(\xi_{p}, t\right), t\right)=\frac{2 \sqrt{Q}}{3}\left(3 \xi_{p}-1\right)+O\left(t^{-1}\right)
\end{aligned}
$$

as $t \rightarrow+\infty$. The results of this section are summarized in Table 1 .

6. Numerics. To illustrate the theoretical results, we next turn to numerical computations. The physical interpretation of the Airy system (1.1) in the presence of shocks requires that it be written in the form of mass-momentum conservation laws (cf. $(3.36)$ )

$$
\eta_{t}+m_{x}=0, \quad m_{t}+\left(\frac{1}{2} \eta^{2}+\frac{m^{2}}{\eta}\right)_{x}=0,
$$

since $m(x, t)=\eta(x, t) u(x, t)$ is the momentum density for the fluid layer of thickness $\eta$. In this form, system (6.1) is mathematically equivalent to (1.1) and preferable for numerical computations with a conservative discretisation scheme that maintains the significance of fluxes for the spatial derivatives above. This can be achieved for (6.1) by means of a shock capturing WENO finite difference scheme [18]. Our specific implementation consists of a standard fifth order WENO method coupled with the fourth order strong stability preserving Runge-Kutta method derived in [20, p. 489] (see also [8]). (We refer to [2] for more details on the shock capturing algorithm adopted in this work.)

Given the presence of a division by the thickness $\eta$ the flux term $m^{2} / \eta$ of this conservative formulation, it is interesting to check how this scheme behaves in the presence of a contact point with a dry/vacuum state when compared with some of 

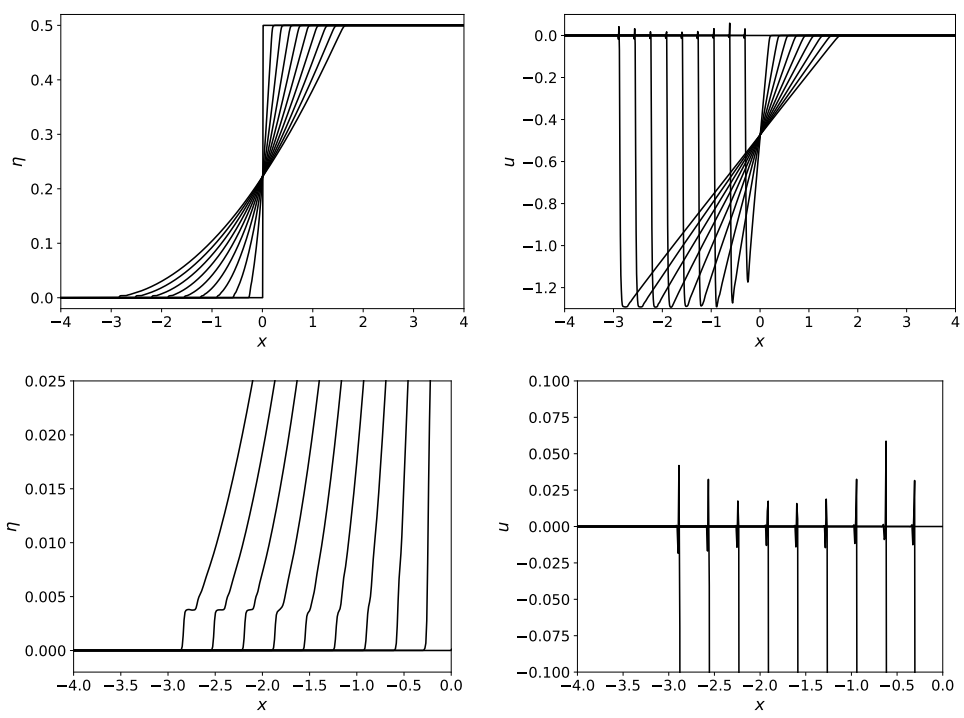

FIG. 10. Riemann dam-break, $Q=1 / 2$. Top row panels: snapshots of evolution of $\eta$ and $u$ from (nondimensional) time $t=0$ to $t=4$ at time intervals 0.4 apart. Bottom row: zoom-in showing shock from wetting layer, and velocity jump with oscillations.

the exact cases we have considered above. Not unexpectedly, the code we have tried breaks down when $\eta=0$ on an interval, and in order to simulate this case it is necessary to extend the surface variable in these dry regions with a thin, but nonzero, wetting layer. Note that the original formulation (1.1) of the Airy system, while free of this division-by-zero problem, is still unsuitable for studying the "vacuum" dam-break problem due to the impossibility of ensuring that the numerical solution conserves momentum.

In Figures 10-12, we show snapshots of the numerical solutions obtained for the initial data discussed in sections 2,3 , and 4 , respectively. For both the linearand the parabolic-connection initial data, we plot the profiles for $\eta$ and $u$ every 0.8 units of time, while for the Riemann problem we double the plotting frequency to 0.4 time units, due to the higher speed of propagation of the rarefaction wave. The regularization value for our computations is $\eta(x<0, t=0)=10^{-6}$.

As seen in the figures, the overall qualitative behaviour of the analytical solution is captured by the scheme, although "fine-tooth-comb" details may differ. The clearest of these differences, shown in the enlargments, consists in a train of high-frequency, unphysical oscillations propagating into the vacuum region to the left of the wetting point. A second inconsistency between the exact solutions and the numerical approximation lies in the (expected) formation of a weak shock wave that appears in the numerical solution as a consequence of the regularisation of the vacuum region. Such a shock wave is physically justified when the low-density region to the left of the dam is not a vacuum state. For this reason, and in contrast to the wiggles described earlier, this shock wave cannot be ascribed to a limitation of the numerical method. However, since the amplitude of the shock tends to zero with the height of the wetting layer, we believe that in our computations the density in the region to the left of the dam is sufficiently low that the perturbed solution follows closely the exact vacuum solution. This limit, however, requires a progressively smaller time step for the algorithm, which affects its performance in the long run. 

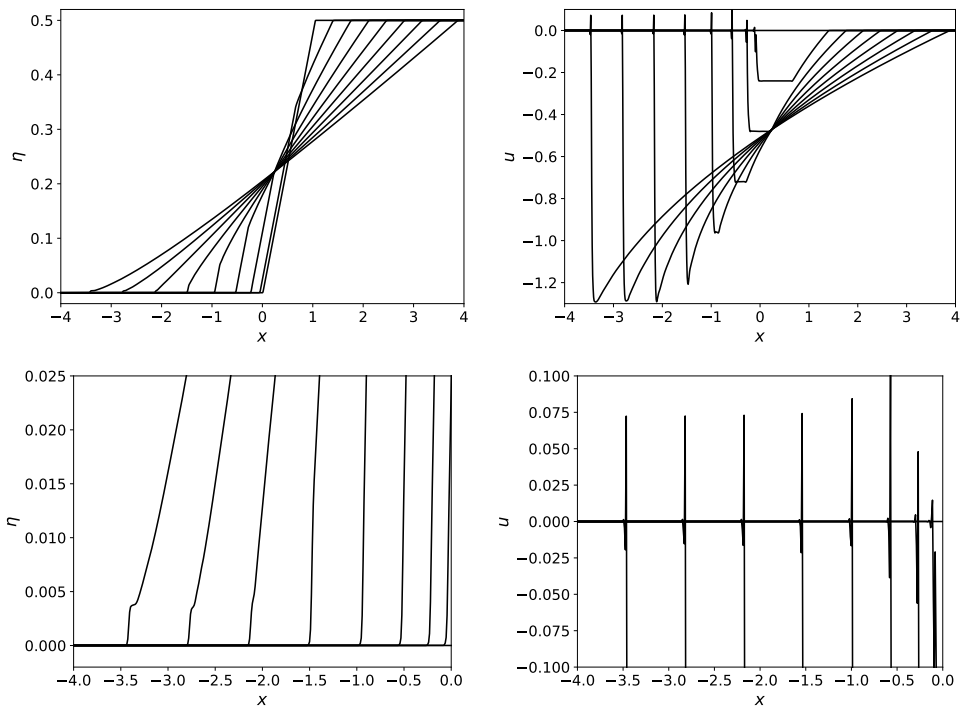

FIG. 11. Linear dam-break, $Q=1 / 2, \beta_{0}=1 / 2$. Top row panels: snapshots of evolution of $\eta$ and $u$ from (nondimensional) time $t=0$ to $t=8$ at time intervals 0.8 apart. Bottom row: zoom-in showing shock from wetting layer and velocity jump with oscillations.
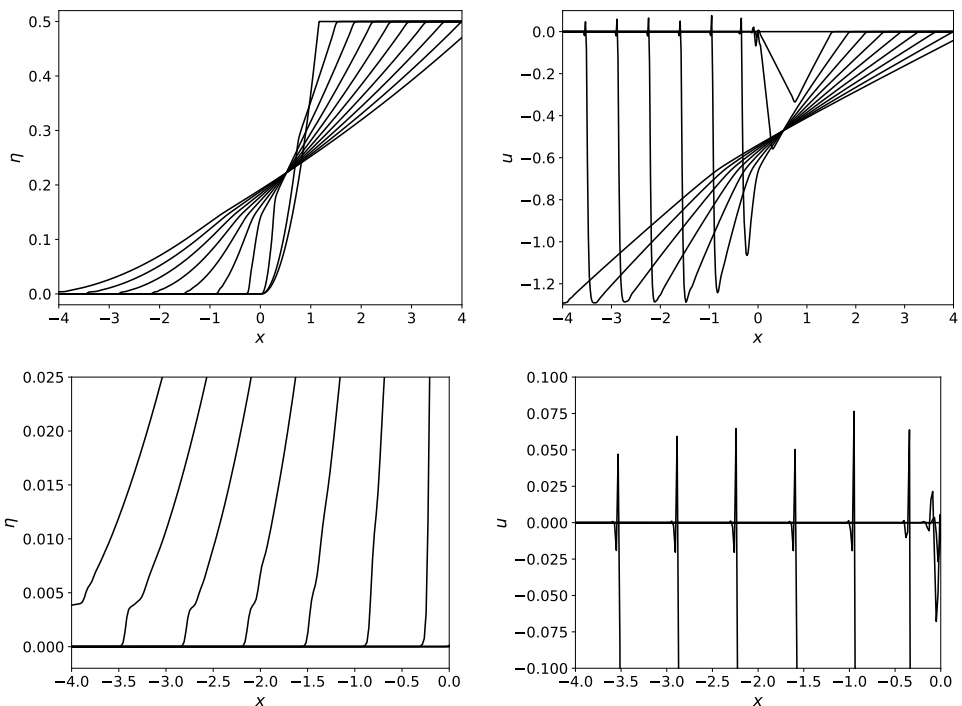

Fig. 12. Parabolic dam-break, $Q=1 / 2, \gamma_{0}=1 / 2$. Top row panels: snapshots of evolution of $\eta$ and $u$ from (nondimensional) time $t=0$ to $t=8$ at time intervals 0.8 apart. Bottom row: zoom-in showing shock from wetting layer and velocity jump with oscillations.

In closing this section, it is interesting to consider, for the classical dam-break of section 2, the regularization offered by the Stoker continuation of the $u$-component of the solution with its constant value $-2 \sqrt{Q}$ for $x<0$. As no shocks are expected to develop, the original dependent variables $\eta, u$ of the Airy system (1.1) can be used, as the solution remains a continuous expansion wave in both dependent variables for 

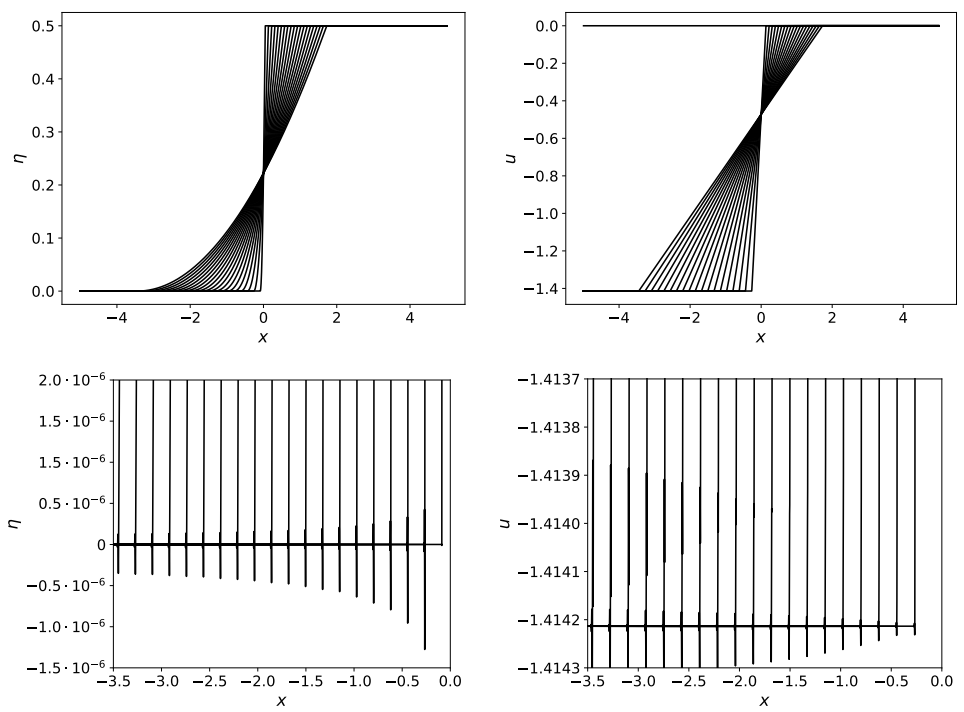

FIG. 13. Riemann dam-break with $u$-continuation $u=-2 \sqrt{Q}$ for $x<0$ with $Q=1 / 2$. Top row panels: snapshots of evolution of $\eta$ and $u$ with from (nondimensional) time $t=0.1$ to $t=8$ at time intervals 0.2 apart. Bottom row: zoom-in showing small (of order $10^{-} 6$ ) initial oscillations around the contact point $p(t)$.

$t>0$. In particular, no regularizing wetting layer $\eta>0$ in the dry region is required to run the code in this case. The result of a computation with the initial data given by (2.6) taking the small initial time $t=0.1$ is shown in Figure 13. This choice of initial time for the initial condition (2.6) effectively smoothes the initial step and avoids a numerical effect of an initial shock near the left-moving front. However, even in this case, a small initial numerical error still occurs and is dissipated away as the evolution proceeds from this initial time.

7. Conclusions. We have considered the classical "dam-break" problem of a fluid layer released onto a dry bed as an illustration of the dynamics supported by gas-dynamic-type equations in the presence of a vacuum. While it might be expected that a rarefaction wave would eventually propagate into the vacuum at long times, at intermediate times the evolution can differ substantially depending on the degree of smoothing regularity of the initial step at the dry point. In fact, the cases we have considered show that the regularity at the vacuum point can change from its initial degree in the course of the evolution. Thus, initial smoothing of class $C$ compactly supported (allowing for jumps in the derivative of the thickness $\eta$ ) as in the case of the linear core example in section 3.2, suggests that this regularity persists for a finite time at the moving front where $\eta=0$. In this case, a simple wave develops and eventually merges at the dry point, thereby increasing its regularity to $C^{1}$, as in the standard dam-break Riemann rarefaction wave. Thus, this case illustrates a mechanism by which the physical vacuum solution breaks down at the finite times when a simple wave overtakes it. In contrast, as in the case of the parabolic core example of section 4 , smoothing of class $C^{1}$ at the dry point pins it down to its initial position (at $x=0$, say) for a finite time, until a gradient catastrophe occurs at time $t=t_{c}$. In our parabolic core example, from the viewpoint of the fluid layer thickness, this catastrophe corresponds to a vertical "wall" of finite extensions that forms for 
the instant $t=t_{c}$ at the dry point. The wall is connected to the quiescent background state by a simple wave solution (the "shoulder") which joins smoothly as a graph to the vertical wall at time $t_{c}$, since the derivative at $x=0$ of the shoulder goes to infinity at that point. From the catastrophe time onward, the wall evolves into a Stoker-like rarefaction wave, which joins in both magnitude and derivative the shoulder. The resulting combined simple wave is a function of $C^{1}$-class, whose support goes from the front to the gluing point with the constant $\eta$ background. At the front, even though the catastrophe destroys the $C^{1}$-class at an instant in time at $t=t_{c}$, this regularity is immediately recovered by the Stoker wave at time $t_{c}^{+}$. As to the long time limit of the solution, we have obtained additional information on its behavior by using a particular form of stretched variables, which allow us to isolate the leading order perturbation terms from the classical Stoker-like rarefaction wave. (Note that compactly supported smoothing of initial data, as in the class we have considered, leads to evolution that can differ significantly from that with smoothing supported on the whole real line, as in the case considered in [7] for a similar problem with the so-called defocusing nonlinear Schrödinger equation.)

The evolution of the velocity field from zero initial data naturally follows suit, being coupled to the thickness $\eta$ by the momentum equation. Thus, we expect $u$ to develop a shock at the front at $t=0^{+}$, as for the linear core case, while for smoothing of class $C^{1}$ at the dry point, as for the parabolic core case, the velocity maintains continuity at the dry point till the catastrophe time. While clearly the velocity of the vacuum is devoid of physical meaning, from the mathematical perspective of the reduction $\eta=0$, the assumption of continuity with respect to initial data leads to fitting a shock for the velocity for the linear core case. These nonstandard, velocity-only, shocks not only satisfy the Rankine-Hugoniot conditions for mass and momentum conservation but would also comply with an infinite class of conservation laws.

Finally, our analysis can be used for testing the performance of popular shockcapturing schemes such as WENO. While the overall features of the solution are well represented by the numerical simulations, there appear to be limitations on the fine details of the moving front, which depend on the formulation being used. Thus, for instance, the density/momentum formulation suffers, for Airy's case, from the division by $\eta$ in the flux, which requires the presence of a thin wetting layer in front of the dam for the numerical method to work. The trend for the wetting layer thickness limiting to zero can be investigated numerically for the initial value problem, but identifying the correct scaling limit can become expensive as it requires increasingly smaller grid sizes. A reformulation of the dam-break as an initial-boundary value problem using thickness and velocity variables helps overcome some of the limitations above by doing away with the need of a wetting layer. However, this requires knowledge of the front position at all times, which cannot be known a priori for general initial dam-break data.

Acknowledgments. R.C. thanks the hospitality of ICERM's program "Singularities and Waves in Incompressible Fluids" in the spring of 2017, when some of this work got underway. R.C., M.P., and G.P. thank the Dipartimento di Matematica e Applicazioni of Università Milano-Bicocca for its hospitality; G.F., G.O., M.P., and G.P. thank the Carolina Center for Interdisciplinary Applied Mathematics at the University of North Carolina for hosting their visits in 2018. All authors gratefully acknowledge the auspices of the GNFM section of INdAM under which part of this work was carried out. Last, but not least, we thank an anonymous referee for bringing the literature on "physical vacuum" to our attention and pointing out the relevance of our results in this context. 


\section{REFERENCES}

[1] R. Camassa, G. Falqui, and G. Ortenzi, Two-layer interfacial flows beyond the Boussinesq approximation: A Hamiltonian approach, Nonlinearity, 30 (2017), pp. 466-491.

[2] R. Camassa, G. Falqui, G. Ortenzi, M. Pedroni, and G. Pitton, Singularity formation as a wetting mechanism in the dispersionless water wave model, Nonlinearity, 32 (2019), pp. 4079-4116.

[3] R. Camassa, G. Falqui, G. Ortenzi, M. Pedroni, and C. Thompson, Hydrodynamic models and confinement effects by horizontal boundaries, J. Nonlinear Sci., 29 (2019), pp. 14451498.

[4] G. Chan And R. C. Young, Shock free solutions of the compressible Euler equations, Arch. Rat. Mech. Anal., 217 (2012), pp. 1265-1293.

[5] S. ChIANG, Non-formation of a vacuum state in a standing piston problem, J. Differential Equations, 230 (2006), pp. 24-28.

[6] D. Coutand AND S. ShKoller, Well-posedness in smooth function spaces for the movingboundary 1-D compressible Euler equations in physical vacuum, Comm. Pure Appl. Math., 64 (2011), pp. 328-366

[7] M. G. Forest And K. T.-R. McLaughlin, Onset of oscillations in nonsoliton pulses in nonlinear dispersive fibers, J. Nonl. Sci., 7 (1998), pp. 43-62.

[8] S. Gottlieb, C.-W. Shu, and E. Tadmor, Strong stability-preserving high-order time discretization methods, SIAM Rev., 43 (2001), pp. 89-112.

[9] A. V. Gurevich And A. L. Krylov, Dissipationless shock waves in media with positive dispersion, Zh. Eksp. Teor. Fiz., 92 (1987), pp. 1684-1699.

[10] F. HuAnG, M. LI, AND Y. Wang, Zero dissipation limit to rarefaction wave with vacuum for 1-D compressible Navier-Stokes equations, SIAM J. Math. Anal., 44 (2012), pp. 1742-1759.

[11] J. JANG AND N. MASMOUdi, Well-posedness of compressible Euler equations in a physical vacuum, Comm. Pure Appl. Math., 68 (2015), pp. 61-111.

[12] J. Kevorkian and J. D. Cole, Perturbation Methods in Applied Mathematics, Springer, New York, (1981).

[13] P. D. LAX, Development of singularities of solutions of nonlinear hyperbolic partial differential equations, J. Math. Phys., 5 (1964), pp. 611-613.

[14] T.-P. LiU, Compressible flow with damping and vacuum, Japan J. Ind. Appl. Math., 13 (1996), pp. $25-32$.

[15] T.-P. Liu And J. A. Smoller, On the vacuum state for the isoentropic gas dynamics equations, Adv. Appl. Math., 1 (1980), pp. 345-359.

[16] Y. Nutku and M. V. Pavlov, Multi-Lagrangians for integrable systems, J. Math. Phys., 43 (2002), pp. 1441-1459.

[17] L. V. Ovsyannikov, Two-layer "shallow water" model, J. Appl. Mech. Tech. Phys., 20 (1979), pp. $127-135$.

[18] C.-W. SHU, High order weighted essentially non-oscillatory schemes for convection dominated problems, SIAM Rev., 51 (2009), pp. 82-126.

[19] T. SIDERIS, Global existence and asymptotic behavior of affine motion of $3 D$ ideal fluids surrounded by vacuum, Arch. Ration. Mech. Anal., 225 (2017), pp. 141-176.

[20] R. J. Spiteri and S. J. Ruuth, A new class of optimal high-order strong-stability-preserving time discretization methods, SIAM J. Numer. Anal., 40 (2002), pp. 469-491.

[21] J. J. Stoker, Water Waves: The Mathematical Theory with Applications, Wiley-Interscience, New York, 1957.

[22] G. B. Whitham, Linear and Nonlinear Waves, Wiley, New York, 1974. 